\title{
Weekly versus biweekly high-dose rate brachytherapy schedules in carcinoma cervix following concomitant chemoradiation with paclitaxel and carboplatin
}

\begin{abstract}
Context: In the management of cervical cancer, though a number of chemotherapeutic drugs have been used for concomitant chemoradiation, paclitaxel and carboplatin have shown improved overall response rates with acceptable side effects. The use of intracavitary irradiation significantly improves survival and reduces local failures. However, the ideal time-dose-fraction schedule remains controversial with high-dose rate (HDR) brachytherapy.

Aims: The aim of this study was to determine the efficacy and safety of paclitaxel and carboplatin for concomitant chemoradiation therapy and to compare the local control, toxicity, and tolerability of the two HDR brachytherapy schedules in patients of carcinoma cervix.

Subjects and Methods: The patients were divided randomly into two groups of thirty patients each. Both the groups were treated with a combination of external beam radiotherapy with $50 \mathrm{~Gy} / 5$ weeks/25 fractions to whole pelvis along with concomitant chemotherapy. The concomitant chemotherapy schedule was injection paclitaxel $\left(135 \mathrm{mg} / \mathrm{m}^{2}\right)$ and injection carboplatin (area under the curve $-4.5 \mathrm{mg} \mathrm{min} / \mathrm{ml}$ ) at 4-week interval followed by HDR brachytherapy. Following which, patients received HDR-intracranial brachytherapy on the basis of random allocation, Group I: 6 Gy $\times 3$ fractions (weekly) and Group II: 5 Gy $\times 4$ fractions (biweekly).

Results and Conclusion: The survival difference between the two groups (weekly and biweekly) was not statistically significant. However, Grade 2-3 rectal reactions in Group I were significantly higher than Group II $(P=0.038)$. The twice-weekly schedule of brachytherapy was a significant factor influencing the late complication rate but not the local pelvic control rate or overall survival rate.
\end{abstract}

Keywords: Cervical cancer, chemoradiation, high-dose rate brachytherapy

\section{INTRODUCTION}

In recent years, various chemotherapeutic agents have been tried for concomitant chemoradiation in carcinoma cervix, either as a single agent or in combination. In an effort to improve the therapeutic ratio, additional chemotherapeutic agents such as carboplatin and paclitaxel have been tested clinically.

Brachytherapy plays a very important role in obtaining high cure rates with minimum complications. High-dose rate (HDR) brachytherapy has experienced tremendous variations in regard to the fractionation schedules. This study was performed to evaluate the efficacy of different

\begin{tabular}{|l|c|}
\hline \multicolumn{2}{|c|}{ Access this article online } \\
\hline \multirow{2}{*}{$\begin{array}{l}\text { Website: } \\
\text { www.asjo.in }\end{array}$} & Quick Response Code \\
\hline \multirow{2}{*}{$\begin{array}{l}\text { DOI: } \\
\text { 10.4103/ASJO.ASJO_111_16 }\end{array}$} & \\
&
\end{tabular}

HDR brachytherapy schedules (6 Gy weekly $\times 3$ and 5 Gy biweekly $\times 4$ ) following concurrent chemoradiation with paclitaxel and carboplatin.

\section{Pooja Gogia, Anil Kumar Dhull ${ }^{1}$, Vivek Kaushal ${ }^{1}$} Department of Radiation Oncology, Andaman Nicobar Islands Institute of Medical Sciences, GB Pant Hospital, Port Blair, Andaman and Nicobar Islands, ${ }^{1}$ Department of Radiation Oncology, Pt. B. D. Sharma Post Graduate Institute of Medical Sciences, Rohtak, Haryana, India

Address for correspondence: Dr. Anil Kumar Dhull, Department of Radiation Oncology, Regional Cancer Centre, Pt. B. D. Sharma Post Graduate Institute of Medical Sciences, P.O. Box 100, GPO, Rohtak - 124 001, Haryana, India. E-mail: anilkdhull@gmail.com

This is an open access article distributed under the terms of the Creative Commons Attribution-NonCommercial-ShareAlike 3.0 License, which allows others to remix, tweak, and build upon the work non-commercially, as long as the author is credited and the new creations are licensed under the identical terms.

For reprints contact: reprints@medknow.com

How to cite this article: Gogia P, Dhull AK, Kaushal V. Weekly versus biweekly high-dose rate brachytherapy schedules in carcinoma cervix following concomitant chemoradiation with paclitaxel and carboplatin. Asian J Oncol 2017;3:12-6. 


\section{SUBJECTS AND METHODS}

Sixty treatment naïve, histopathologically proven Stage I-III patients of carcinoma cervix having Karnofsky performance scale (KPS) $\geq 70$ and normal hematological parameters were included in the study. Patients who had prior radiation, surgery or chemotherapy, and general condition too poor with KPS $<70$ were excluded from the study.

The pretreatment evaluation included history, complete systemic examination including gynecological examination, hematological and biochemistry studies, chest radiography, and ultrasonography of abdomen and pelvis. A computed tomography scan/magnetic resonant imaging scan of abdomen and pelvis were performed only when clinically indicated.

All sixty patients were treated with a combination of external beam radiotherapy (EBRT) $50 \mathrm{~Gy} / 5$ weeks/25 fractions to whole pelvis along with concomitant chemotherapy in both the groups. The patients were planned with "four-field box" technique. The patients received the concomitant chemotherapy schedule with injection paclitaxel $\left(135 \mathrm{mg} / \mathrm{m}^{2}\right)$ and injection carboplatin (area under the curve [AUC] - $4.5 \mathrm{mg}$ $\mathrm{min} / \mathrm{ml}$ ) at 4-week interval on day 1 and 28 of external radiation.

The patients were randomly divided into two groups of 30 patients each by draw of lots for receiving HDR brachytherapy. Group I (weekly) patients received brachytherapy by HDR-intracavitary brachytherapy (ICBT) -6 Gy $\times 3$ fractions given weekly, whereas Group II patients (biweekly) received brachytherapy by HDR-ICBT -5 Gy $\times 4$ fractions, 2 fractions per week, with brachytherapy administered on biweekly basis.

Disease status was evaluated according to the WHO criteria, and treatment-related complications and late toxicities were evaluated according to the Radiation Therapy Oncology Group (RTOG)/European Organization for Research and Treatment of Cancer late toxicity criteria. The patients were assessed for any evidence of distant metastasis during each follow-up visit.

Statistical analysis was carried out using Stata 9.0 (IBM SPSS Statistics version 16.0, Chicago, IL, United States) and variables were compared using Chi-square test/Fisher's exact test, and quantitative characteristics were compared using Student's $t$-test for independent samples/Wilcoxon rank-sum test.

\section{RESULTS}

The median age (range) at presentation in Group I and II was 52.5 (35-70) and 55 (38-75) years, respectively. All patients' characteristic data were comparable in both the groups.
Acute toxicity profile during chemoradiation

The side effects of concomitant chemoradiation therapy during four weekly paclitaxel and carboplatin were graded as per RTOG criteria: three patients in each group developed Grade 3-4 upper gastrointestinal (GI) reactions; six patients in Group I and five in Group II developed acute Grade 3-4 lower GI reactions; six patients in Group I and four patients in Group II developed acute Grade 3-4 cutaneous reactions; and four patients in each group developed Grade 3 mucosal toxicity whereas one patient in each group developed Grade 3-4 hematological toxicity. None of the patients developed Grade 3 genitourinary toxicity in either group. The acute toxic reactions between both the groups were not statistically significant.

The acute toxicities leading to interruption of chemoradiation treatment in Group I and II were as follows: Grade 2-3 GI toxicity $3 / 30(10 \%)$ each in both the groups, Grade 3 skin reactions $1 / 30(3 \%)$ and $2 / 30(7 \%)$, and Grade 3 hematological toxicity $0 \%$ and $(2 / 30) 7 \%$, respectively.

A number of patients who completed intended chemoradiation treatment without interruption in Group I and II were 87\% and $77 \%$, respectively. All patients tolerated treatment with minor morbidity. Major acute toxicities were rarely seen.

\section{Local control rate and disease-free survival rate}

Local control in Group I and II was similar and, respectively, was as follows: complete response (CR) - 26/30 (87\%) and partial response (PR) - 4/30 (13\%). All the patients were suitable for ICBT.

Follow-up in our patients ranged from 6 to 18 months. Survival was analyzed with multivariate analysis. Disease-free survival (DFS) in Group I and II was $83 \%$ and $80 \%$, respectively. The survival difference between the two groups was not statistically significant $(P=0.782)$.

At the last follow-up, $7 \%$ of patients had residual disease in Group I (Stages IIA and IIIB) and 7\% of patients had residual disease in Group II (both Stage IIIB). One patient of Group I had recurrent disease (Stage IIIB) and one patient in Group II (Stage IIB) had recurrent disease. The DFS was not influenced by age, morphology of tumor, histopathology, and tumor size.

\section{Late toxicity profiles}

The side effects of late radiation therapy (RT) were graded as per RTOG criteria. Late reactions and their grades in Group I and II were as follows - bladder toxicity: grade $1(3 / 30) 10 \%$ in both the groups and Grade $2(1 / 30) 3 \%$ versus $(0 / 30) 0 \%$; rectal toxicity: Grade $1(3 / 30) 10 \%$ in both the groups, Grade $2(3 / 30)$ 
Gogia, et al.: HDR brachytherapy schedules in carcinoma cervix

10\% versus nil, and Grade 3 (1/30) 3.3\% versus nil; cutaneous radiation reactions: Grade 1 (17/30) 57\% versus (12/30) 40\%, Grade 2 nil; and vaginal toxicity: Grade 1 (9/30) 30\% versus (10/30) 33.3\% and Grade 2 (8/30) 27\% versus (4/30) 13\%. Although the late radiation reactions in Group I were higher than Group II, they were not statistically significant. However, Grade 2-3 proctitis in Group I was significantly higher than Group II $(P=0.038)$.

\section{DISCUSSION}

Cancer of the uterine cervix is the leading cause of cancer mortality and is the second most common cancer in women after breast cancer in India. ${ }^{[1,2]}$ Radical radiotherapy fails to control $35 \%-85 \%$ of patients with locally advanced cervical cancer. Simultaneous radiochemotherapy has demonstrated to be superior to radiation alone. ${ }^{[3]}$

Recent studies have shown a better response with the addition of newer chemotherapeutic agents such as paclitaxel and carboplatin. With its cytotoxic and radiosensitization effect, it reduces local and distant recurrences in patients with cervical cancer when used concomitantly with RT in carcinoma cervix are associated with significantly improved overall response rates and acceptable toxicity. ${ }^{[4-7]}$

In view of the therapeutic ratio, cumulative biological effective dose (BED) 70-80 Gy (10) at point A is appropriate for uterine cervical cancer patients treated with a combination of EBRT and HDR-ICBT. The present results and data from other literatures suggested that cumulative BED at the rectal point should be kept below 100-120 Gy (3) to prevent late rectal complication. ${ }^{[8]}$

However, there is no consensus as to the best dose-fractionation regimen in HDR brachytherapy for cervical cancer. A number of different HDR fraction numbers, from 2 to 15 , are currently in use. It would be predictable on radiobiological grounds, and it is certainly observed in clinical practice, that if doses are matched for early effects, a protocol consisting of a few large fractions will result in worse late effects than a protocol consisting of many small fractions or continuous low-dose rate. ${ }^{[9]}$ Our study compared the effectiveness of the two HDR brachytherapy schedules which have similar BED in locally advanced cervical carcinoma that was treated with concurrent chemoradiotherapy. Patients were divided into Group I: 6 Gy $\times 3$ fractions (weekly) and Group II: 5 Gy $\times 4$ fractions (biweekly).

In the present study, the local control at the end of chemoradiation was carefully documented and observed that
$\mathrm{CR}, \mathrm{PR}$, and overall response in Group I and II were similar without any statistically significant difference. A total of eight patients (13\%) had PR and rest showed a CR.

Eighty-two percent of the patients completed intended treatment without interruption with Grade 2-3 GI toxicity being the major cause of treatment interruption (10\% each in both the groups).

Similar results were reported by Addeo et al., where they conducted a study of combining RT with twice-weekly paclitaxel $\left(30 \mathrm{mg} / \mathrm{m}^{2}\right)$ and carboplatin (AUC of 6), achieved a clinical response in eight patients $(89 \%)$ and with a CR in five patients (56\%). Two out of the five patients who received carboplatin at AUC 6 developed Grade 3 toxicity. $^{[4]}$

Furthermore, Higgins et al. conducted a study to determine the feasibility of weekly carboplatin/paclitaxel with RT in the primary treatment of cervical cancer. Carboplatin (mean dose $245 \mathrm{mg}$ ) and paclitaxel (mean dose $70 \mathrm{mg}$ ) were successfully administered in $97 \%$ and $90 \%$ of planned treatments, respectively. Grade 3/4 hematologic or GI toxicity was unusual. The CR rate 3 months after completion of therapy was $91 \% .{ }^{[6]}$

Thus, chemoradiation with paclitaxel and carboplatin showed encouraging results with acceptable toxicity.

In our study, the survival difference between the two groups (weekly and biweekly) was not statistically significant $(P=0.7823)$. Results in terms of locoregional control and DFS in both the groups are consistent with that reported in literature.

A clinical trial by Le Pechoux et al., where external beam irradiation to the pelvis (mean dose of $50 \mathrm{~Gy}$ ), combined with HDR brachytherapy (mean dose of 20 Gy to point A) given 5 Gy per session twice weekly or 6 Gy once weekly in carcinoma cervix. Local recurrence rate was $11 \%$ and distant failure rate was $21 \%$, with no difference between the two groups. ${ }^{[10]}$

A study from Thailand compared the effectiveness of the two HDR brachytherapy schedules in locally advanced cervical carcinoma that was treated with concurrent chemoradiotherapy. With a median follow-up time of 35 months, there was no statistical significance in terms of local control, DFS, and overall survival and complication rates between the two treatment schedules which could be observed. ${ }^{[1]}$ 
Gogia, et al.: HDR brachytherapy schedules in carcinoma cervix

A similar study was conducted to study for late effects after radiotherapy for locally advanced cervical cancer and comparison of two brachytherapy schedules. No significant difference was found between the two groups. ${ }^{[12]}$

All the late radiation reactions in Group I were higher than Group II though not statistically significant. However, Grade 2-3 rectal reactions in Group I were significantly higher than Group II $(P=0.038)$. These observations are similar to that reported by other workers.

Similarly, Huang et al. conducted a prospective study to compare treatment results between two fractionation schedules of HDR-ICBT, patients underwent either $6 \mathrm{~Gy} \times 4$ (once weekly) or $4.5 \mathrm{~Gy} \times 6$ (twice weekly) to point $A$. The rates of overall survival, locoregional failure, distant metastasis, cystitis, and enterocolitis were not significantly different between HDR-4 and HDR-6 schedules. The corresponding rates of Grade 2-4 proctitis were $18.7 \%$ and $9.6 \%(P=0.060)$. The corresponding rates of Grade 3-4 proctitis were $5.2 \%$ and $1.3 \%(P=0.231) .{ }^{[13]}$

In another study, 541 women diagnosed with cervical cancer were treated with curative-intent radiotherapy. Multivariate analysis revealed that the fractionation scheme ( 3 fractions vs. 5 fractions) was a significant factor influencing the proctitis rate but not the local pelvic control rate, overall survival rate, or cystitis rate. ${ }^{[14]}$

Hama et al. conducted a study comparing once- versus twice-weekly HDR brachytherapy in carcinoma cervix patients. One hundred and twenty-four consecutive previously untreated patients with cervical cancer were treated with external beam irradiation (50 Gy) and HDR brachytherapy. A total of 74 patients (Group I) were treated with HDR brachytherapy once weekly (about 3 fractions of 7 Gy each to point A) whereas 50 patients (Group II) were treated twice weekly (about 6 fractions of 4.5 Gy each to point A). In conclusion, the twice-weekly HDR regimen may improve the local control rate with fewer complications than once a week HDR regimen. ${ }^{[15]}$

\section{CONCLUSION}

The management of patients with carcinoma cervix using four weekly concomitant chemoradiation with paclitaxel and carboplatin is feasible with good pelvic control and with acceptable toxicities. The twice-weekly schedule of brachytherapy was a significant factor influencing the late complication rate but not the local pelvic control rate or overall survival rate.

\section{Acknowledgment}

The authors would like to thank the Department of Radiation Oncology, Regional Cancer Centre, Pt. B. D. Sharma Post Graduate Institute of Medical Sciences, Rohtak - 124 001, Haryana, India.

\section{Financial support and sponsorship}

Nil.

\section{Conflicts of interest}

There are no conflicts of interest.

\section{REFERENCES}

1. Viswanathan AN. Uterine cervix. In: Halperin EC, Wazer DE, Perez CA, Brady LW, editors. Principles and Practice of Radiation Oncology. $6^{\text {th }}$ ed. Philadelphia: Lippincott Williams and Wilkins; 2013. p. $1355-424$.

2. Ferlay J, Soerjomataram I, Ervik M, Dikshit R, Eser S, Mathers C, et al. GLOBOCAN 2012, Cancer Incidence and Mortality Worldwide: IARC Cancer Base No. 11. Lyon, France: International Agency for Research on Cancer; 2013. Available from: http://globocan.iarc.fr. [accessed on 2015 Jan 23].

3. Mountzios G, Soultati A, Pectasides D, Dimopoulos MA, Papadimitriou CA. Novel approaches for concurrent irradiation in locally advanced cervical cancer: Platinum combinations, non-platinum-containing regimens, and molecular targeted agents. Obstet Gynecol Int 2013;2013:536-765.

4. Addeo D, Blank S, Muggia F, Formenti S. Concurrent radiotherapy, paclitaxel and dose escalating carboplatin in the treatment of cervical cancer - A phase I study. Anticancer Res 2008;28:3143-6.

5. Lee MY, Wu HG, Kim K, Ha SW, Kim JS, Kim IA, et al. Concurrent radiotherapy with paclitaxel/carboplatin chemotherapy as a definitive treatment for squamous cell carcinoma of the uterine cervix. Gynecol Oncol 2007;104:95-9.

6. Higgins R, Bussey M, Naumann W, Hall J, Tait D, Haake M, et al. Concurrent carboplatin and paclitaxel with pelvic radiation therapy in the primary treatment of cervical cancer. Gynecol Oncol 2007;89:499-503.

7. de Vos FY, Bos AM, Gietema JA, Pras E, Van der Zee AG, de Vries EG, et al. Paclitaxel and carboplatin concurrent with radiotherapy for primary cervical cancer. Anticancer Res 2004;24:345-8.

8. Toita T, Kakinohana Y, Ogawa K, Adachi G, Moromizato H, Nagai Y, et al. Combination external beam radiotherapy and high-dose-rate intracavitary brachytherapy for uterine cervical cancer: Analysis of dose and fractionation schedule. Int J Radiat Oncol Biol Phys 2003;56:1344-53.

9. Hall EJ, Brenner DJ. The dose-rate effect revisited: Radiobiological considerations of importance in radiotherapy. Int J Radiat Oncol Biol Phys 1991;21:1403-14.

10. Le Pechoux C, Akine Y, Sumi M, Tokuuye K, Ikeda H, Yajima M, et al. High dose rate brachytherapy for carcinoma of the uterine cervix: Comparison of two different fractionation regimens. Int J Radiat Oncol Biol Phys 1995;31:735-41.

11. Tharavichitkul E, Klunkin P, Lorvidhaya V, Sukthomya V, Chakrabhandu S, Pukanhaphan N, et al. The effects of two HDR brachytherapy schedules in locally advanced cervical cancer treated with concurrent chemoradiation: A study from Chiang Mai, Thailand. J Radiat Res 2012;53:281-7.

12. Hellebust TP, Kristensen GB, Olsen DR. Late effects after radiotherapy for locally advanced cervical cancer: Comparison of two brachytherapy 
schedules and effect of dose delivered weekly. Int J Radiat Oncol Biol Phys 2010;76:713-8.

13. Huang EY, Sun LM, Lin H, Lan JH, Chanchien CC, Huang YJ, et al. A prospective cohort study to compare treatment results between 2 fractionation schedules of high-dose-rate intracavitary brachytherapy (HDR-ICBT) in patients with cervical cancer. Int J Radiat Oncol Biol Phys 2013;85:123-8.
14. Wang CJ, Huang EY, Sun LM, Chen HC, Fang FM, Hsu HC, et al. Clinical comparison of two linear-quadratic model-based isoeffect fractionation schemes of high-dose-rate intracavitary brachytherapy for cervical cancer. Int J Radiat Oncol Biol Phys 2004;59:179-89.

15. Hama Y, Uematsu M, Nagata I, Shioda A, Suda A, Sakurai Y, et al. Carcinoma of the uterine cervix: Twice- versus once-weekly high-dose-rate brachytherapy. Radiology 2001;219:207-12. 